\title{
Dental biomaterials: challenges in the translation from lab to patient
}

\author{
SADJ February 2020, Vol. 75 No. 1 p16 - p28
}

E Patel' ${ }^{1}$, YE Choonara ${ }^{2}$, V Pillay ${ }^{3}$

\begin{abstract}
Biomaterials are essential components of modern medicine. Dental biomaterials include any material or device used within the oral cavity for the diagnosis and treatment of oral conditions, diseases, and disorders.

Whilst the literature remains abuzz with innovative and diverse technologies, an apparent disconnect is evident in the follow-through, affecting the impact on current clinical treatment regimens.
\end{abstract}

This review will explore the product development process from concept to clinical application, in conjunction with the commercial aspects that affect clinical translation.

Though serving the purpose of a roadmap for novice inventors, the primary intention of the paper is to focus on some of the challenges cited in the literature and to highlight factors that delay or, in due course, actually prevent clinical translation of even the grandest inventions.

Clinicians should also be alert to the complexities affecting the arrival in their surgeries of new products and technologies, The ultimate aim is to assist in the decision-making of researchers when they may be initiating novel advances in oral-related therapies, by ensuring they are cognisant of past errors and limitations, whilst at the same time recognizing present hurdles.

\section{Keywords}

Biomaterials, challenges, clinical translation, dental biomaterial challenges, biomaterial commercial challenges.

\section{Author affiliations:}

1. Ebrahim Patel: BDS, MSc (Dent), Department of Paediatric \& Restorative Dentistry, School of Oral Health Sciences, Faculty of Health Sciences, University of the Witwatersrand, South Africa. ORCID Number: 0000-0002-7683-4867

2. Yahya E Choonara: B.Pharm, M.Pharm, PhD, Wits Advanced Drug Delivery Platform Research Unit, Department of Pharmacy and Pharmacology, School of Therapeutic Sciences, Faculty of Health Sciences, University of the Witwatersrand, South Africa. ORCID Number: 0000-0002-3889-1529

3. Viness Pillay: B.Pharm, M.Pharm, PhD, Wits Advanced Drug Delivery Platform Research Unit, Department of Pharmacy and Pharmacology, School of Therapeutic Sciences, Faculty of Health Sciences, University of the Witwatersrand, South Africa. ORCID Number: 0000-0002-8119-3347

Corresponding author: Ebrahim Patel

Department of Paediatric \& Restorative Dentistry, School of Oral Health Science, 7 York Road, Parktown, Johannesburg,2193, South Africa. Email: ebrahim.patel@wits.ac.za

Author contributions:

1. Ebrahim Patel: First author - $70 \%$

2. Yahya E Choonara: Second author - $15 \%$

3. Viness Pillay: Third author $-15 \%$

\section{ACRONYMS AND FORMULAE}

CaP: $\quad$ Calcium Phosphate, $\mathrm{Ca}_{3} \mathrm{PO}_{4}$

PPA: $\quad$ Provisional Patent Application

RPA: $\quad$ Regular Patent Application

USPTO: US Patents and Trademarks Office

\section{INTRODUCTION}

Biomaterials have become essential components in modern medicine. Advancements in medical technology have led to an updated definition, which, according to the journal Biomaterials (Elsevier), may be quoted as: "any substance that is engineered to take a form, which either alone or as part of a complex system, interacts with components of living tissue to direct the course of any therapeutic or diagnostic procedure".

In general, biomaterials are categorized as being either bioinert, bioactive or biodegradable. Bioinert materials do not interact with the tissue/environment in which they are placed e.g. bone screws and plates. In contrast, bioactive materials directly interact with their surrounding environment. Such interactions include those in which the material binds chemically to hard or soft tissue, those which induce the release of a biological substance, or those improving the healing ability of a tissue, etc. The objective of a biodegradable material is to offer distinct advantages for a limited time period. A typical example would be suture materials that degrade at a rate similar to tissue regeneration. ${ }^{1,2}$

There are five major classes of biomaterials; namely: polymers, metals, bioceramics, natural materials, and composites. ${ }^{3}$

\section{Polymers}

Polymers represent the largest class of biomaterials. They may be harvested from natural resources (such as plant and animal materials) or synthesized in a laboratory. Examples of polymers derived from plant material include cellulose and sodium alginate, whereas polymers derived from animals include collagen, hyaluronic acid and heparin. Synthetic polymers are produced by the co-polymerization of conventional monomers. Due to the nature of their synthesis, they can be manipulated to suit almost any environment.

These materials can be biocompatible, hydrophobic or hydrophilic, biodegradable or non-resorbable, and so forth. Their versatility allows for structural changes to occur upon introduction to a biological environment, which is why they are a favoured component of 
controlled drug release systems. Examples of synthetic polymers include polyamides, poly $(D, L$-lactideco-glycolide) and polyethylene. ${ }^{4}$

\section{Metals}

Metallic biomaterials have been used in medical treatment for over a century. Whilst Lane in 1895 reported the use of metallic plates for fixation of bone fractures, there is archaeological evidence of gold being used in dentistry as early as 1600 BC in Egypt.5,6 Examples of metal biomaterials include stainless steel, titanium, aluminium and cobalt-chromium (Co-Cr).

The use of these materials has gained much popularity in the fields of Orthopaedics and Dentistry. Biomedical applications in these fields include dental implants, dental prosthetics (denture, crown and bridgework), hard tissue replacements (artificial joints) as well as cardiac implants (artificial vascular stents).

Whilst the metals remain bioinert in most cases, surface modifications (for example, those in dental implants) allow for interaction with the biological system making them bioactive. ${ }^{7,8}$

\section{Bioceramics}

Bioceramics refers to the use of specifically designed ceramics for the reconstruction and repair of damaged or diseased areas of the body. They may be bioinert (e.g. aluminium and zirconia), biodegradable (e.g. tricalcium phosphate) or bioactive (e.g. hydroxyapatite and bioactive glass). Bioceramics are employed in Dentistry for periodontal treatment, endodontic treatment, and maxillofacial reconstruction. Their use in orthopaedics includes, but is not limited to, the treatment/replacement of hips, knees, tendons and ligaments. ${ }^{9,10}$

\section{Natural materials}

Natural biomaterials are further classified as: proteinbased (collagen, gelatin), polysaccharide-based (cellulose, chitosan) and tissue-derived (decellularized heart valves, blood vessels) materials. They offer distinct advantages over the synthetic materials, including biocompatibility, biodegradability and remodelling.

In addition, they offer prospects of functioning at a molecular level, as opposed to the macroscopic level. However, due to their complex structure, more intricate strategies are often required for their manipulation to achieve the desired function. ${ }^{11,12}$

\section{Composites}

Composites refer to biomaterials that contain two or more constituent materials or distinct phases. They are either synthetic (such as dental composite filling materials) or natural (such as bone). They may also be either particulate or fibrous, or both, in nature.

Similar to polymers, a major advantage of composites is the ability to manipulate the manufacturing process to yield the desired material properties. These materials can be employed in both hard and soft tissue applications including: dental restorations, bone fracture repair, joint replacement, wound dressings, implants and grafts. ${ }^{4,8}$

\section{The product development process}

The development of a novel biomaterial requires several steps prior to its use in clinical treatment.

Figure 1 outlines a generic timeline from conceptualization to application in patient treatment.

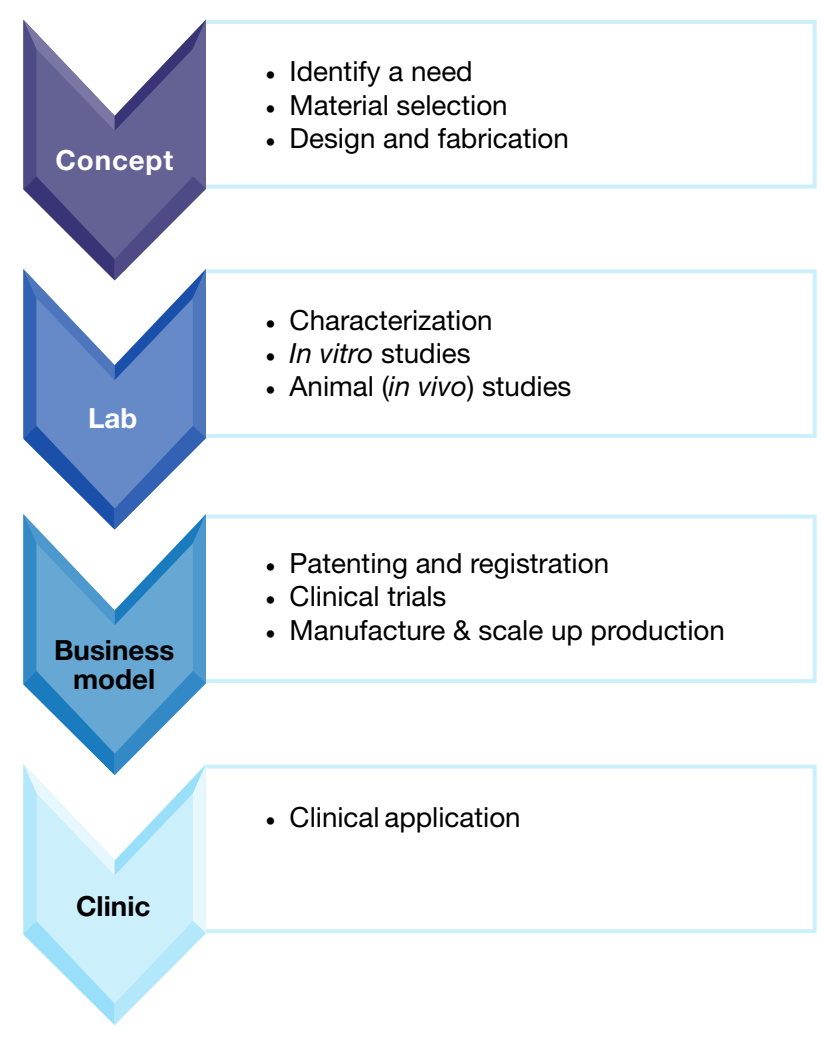

Figure 1. Schematic representation of the translation process of the prefinal version of SA POQL tool.

\section{Concept}

\section{a. Identifying a need}

The conceptualization of a novel biomaterial is the foundation of the development journey. To identify a need within a specific treatment modality, or to recognize a gap within the current literature, the researcher/scientist requires a certain level of expertise in the chosen field of study.

The creativity and open mindedness of the researcher at this initial phase is conceivably the greatest limiting factor. Great inventions are reliant on the ideas and ambitions of workers in the field, who identify and explore new areas to improve and augment current medical strategies. An additional factor that hinders the beginning of the journey is the perceived disconnect between clinician and scientist.

Often we find that the clinician is in fact the scientist and it is in those cases that many great ideas are conceptualized. Whilst scientists possess the knowledge of materials and their capabilities, it is the clinicians who will recognize shortcomings within their respective fields. Collaborations are thus important to yield discussions that will cultivate innovative strategies for improved and enhanced patient care. 


\section{b. Material Selection}

The dynamic environment of the oral cavity requires a concerted effort from oral biologists in the unveiling of its intricate mechanisms. Biomedical engineers attempt to replicate these processes using biomaterials.

Clinicians then assist in the translation of these materials to patient treatment. ${ }^{13}$ In the hope to hasten clinical application, scientists often choose to use for their novel inventions constituents which had been previously approved by regulators.

There are stringent criteria governing the selection of constituents which may be incorporated in dental biomaterials. These criteria stem from the expectations of functions in a complex environment. Materials exposed to the oral cavity are constantly bathed by saliva and its components including enzymes (salivary amylase) and immunoglobulins $(\lg A)$. Sporadic thermal changes may also occur during mastication when ingested food and liquids come into contact with these materials.

Periodontal materials are bathed by crevicular fluid and possibly constituents of the inflammatory process (should the tissue be injured by disease or otherwise). Whilst materials used in endodontic treatment are protected from the effects of the oral cavity at large, they function in an anaerobic environment with little or no fluid.

This affects biodegradeable materials which usually require a catalyst to drive their degradation. An additional subset of dental biomaterials includes those used within bone such as dental implants. These materials are subject to innate and humoral immune responses and often come in close contact with adjacent blood vessels.

Nanotechnology is one of the cornerstone achievements of dental biomaterials. Dental nanomaterials offer several functions including: antimicrobial activity, mechanical reinforcement, aesthetics and therapeutic effects. ${ }^{14,15}$ Nanoparticles such as silver, zinc oxide and titanium dioxide are utilized in endodontic, restorative and implant treatments for their antibacterial properties.

Other nanoparticles such as gold have been used as radiosensitizers, photothermal agents and drug delivery carriers. Apprehension around these materials usually stems from technical challenges (related to agglomeration that hinders expected properties) and a failure to fully understand their possible toxicity in vivo.

An important example of a dental nanomaterial is calcium phosphate, $\mathrm{Ca}_{3} \mathrm{PO}_{4}(\mathrm{CaP})$. $\mathrm{CaP}$ is often utilized for dental applications due to its biocompatibility, bioactivity and composition which is comparable to mineralized tissue (bone and teeth). A major component of natural mineralized tissue is carbonate apatite which is a form of CaP. ${ }^{16}$

Whilst CaP's are osteoconductive, they lack the ability to induce bone de novo. Thus, periodontal and maxillofacial applications for these materials require the addition of bioactive proteins, growth factors and osteogenic drugs to enable inductive properties. ${ }^{17}$
The particle size of the CaP's must also be accounted for. Particles exceeding $200 \mathrm{~nm}$ in size may lead to excessive entry of calcium ions in to cells endangering homeostasis. The challenge to maintain these $\mathrm{CaP}$ nanoparticle sizes occurs following drug incorporation where maintenance of the size within range is difficult. ${ }^{17}$ As previously described, polymers represent the largest class of biomaterials and have been employed in several materials. Natural polymers such as chitosan, hyaluronic acid, alginate, fibrin and collagen etc. are derived from natural sources. A major advantage of this subgroup is their biocompatibility and biological activity which is often manipulated in tissue engineering for cell adhesion and growth. ${ }^{18}$

The shortcomings of natural materials relate to their low mechanical strength which makes them difficult to engineer, and restricts processing and manufacturing capabilities. In addition, batch variability and the risk of pathogen transmission negatively impact decisions to use this polymer subgroup. ${ }^{13}$

Synthetic polymers have overshadowed the natural materials due to their apparent advantages including well-defined chemistry and formulations, ease of processing, tunable degradation kinetics and functionspecific manipulation. ${ }^{19}$ Then again, the synthetic variants are not inherently biocompatible and often provoke unsolicited inflammatory responses.

The magnitude of this inflammatory response is often driven by the heterogeneity, surface topography, and physical and chemical properties of the material. ${ }^{1}$ Efforts to overcome this challenge are usually linked to the attachment of biomolecules to reduce the cytotoxicity of the material. ${ }^{20}$ One such example is the addition of sodium phosphorylated chitosan to reduce the cytotoxicity of zinc oxide nanoparticles.

An additional group of polymers that have gained popularity, with a greater intensity observed in associated research, are "smart" or "intelligent" polymers. Smart polymers offer trigger-related drug delivery and are sensitive to changes in temperature, light, $\mathrm{pH}$, magnetic field and ionic strength. ${ }^{21-23}$ Smart polymers that have a low critical solution temperature are valuable drug delivery agents for fever and/or local infections and are therefore employed as thermo-sensitive biomaterials.

A prior challenge for thermo-sensitive polymers was to create a biodegradable material that functioned (with a release of the drug) at just above normal body temperatures.

This requirement was supposedly solved, with excellent prospects for thermo-sensitive polymers. ${ }^{24}$ However, the intra-oral applications for these materials may still be limited in areas of poor thermal control (e.g. the dental root canal space).

Similarly, light-activated smart polymers require the patient to maintain the device in a dark environment to prevent premature drug release. This phenomenon has been termed "dark toxicity" and is an additional concern for light activated smart polymers. ${ }^{25}$ 


\section{Design and fabrication}

Once the appropriate materials have been chosen for incorporation into a novel biomaterial or drug delivery device, the scientist must then decide on a suitable design and method of fabrication. Whilst the particular methods of synthesis may be generic for most materials, the clinical requirements often guide deviations from the norm. Moghanjoughi et al. listed some of the disadvantages of controlled drug delivery systems that hinder clinical translation. ${ }^{23}$

Amongst the factors were possible dose dumping, cytotoxicity, delayed onset of action, increased risk of hepatic first-pass metabolism, high manufacturing costs, and surgical procedures which may be required for insertion or removal of the device/material. To counter these factors, the design and fabrication process often requires remodelling to yield the desired clinical results. However, manufacturing costs may then increase, placing further strain on the possibility of clinical translation.

Fabrication methods are based on the functions for which the material is intended. Examples of oral drug delivery devices include: tablets, sprays, mouthwashes, gels, patches, pastes and wafers/films. These formulations are dependent on trans-mucosal delivery of the active constituent and must offer practicality, adequate drug release, and most importantly, patient acceptability. Drug release is directly dependent on the ability to penetrate the oral mucosa's epithelium, whilst factors that deter patients from accepting a new drug include disturbances to eating, taste and speech. ${ }^{26}$ More recent technologies to aid trans-mucosal delivery include permeability enhancers, absorption enhancers, enzyme inhibitors, drug delivery vectors (liposomes \& polymerosomes) and muco-adhesives.

Muco-adhesive drug delivery systems present an additional domain in fabrication methods. Within the confines of the oral cavity, these systems include: mucosal patches, films, gels and ointments. These systems boast many advantages over their systemic counterparts including; prolonged adhesion that enhances absorption (further amplified by the blood circulation of the region), a faster onset of action, increased drug bioavailability due to no first-pass metabolism, decreased drug degradation in the acidic GIT environments and improved patient compliance. ${ }^{26,27}$ However, they are not exempt from the complexities of the oral environment. Differences in the degree of keratinization and epithelial thicknesses across the varying mucosal surfaces lead to diverse permeabilities which in turn affect drug absorption rates and bioavailability.

The buccal mucosa is considerably less permeable than the sublingual mucosa, which decreases absorption and bioavailability. On the other hand, the sublingual mucosa is uneven, mobile and constantly washed by saliva making it an unsuitable area for muco-adhesion. ${ }^{28}$ A compromise is thus made with current systems that utilize the buccal mucosa. Novel inventions must account for these shortcomings if they are to improve current therapeutic standards.
Fabrication methods incorporating surface modifications of biomaterials to improve properties such as biointegration have gained momentum. Padovani et al. described the possible difficulties experienced whilst optimizing the concentration of nanoparticles at the implant surface. ${ }^{15}$ They inferred that an overactive rate of drug of nanoparticle/drug release could produce toxicity that would result in necrosis of healthy cells.

Furthermore, León \& Jansen described the non-uniform deposition of CaP coatings on bone screws when techniques such as sputter deposition, pulsed laser deposition and ion beam deposition were used. ${ }^{29} \mathrm{~A}$ recent method, biofabrication, attempts to incorporate cells into biomaterial constructs that enhance cellular attachment, migration and growth.

Biofabrication may also serve as a method for controlled drug delivery and release. However, at present, there is no engineered substitute generated by such biofabrication techniques that can be scaled up for clinical use. ${ }^{30}$

Larry Hench, the father of modern bioactive glass and eramics, admitted that whilst dozens of ceramic compositions are tested in vitro, few ever achieve clinical application. ${ }^{31} \mathrm{He}$ further explained that the clinical success of these materials requires the simultaneous achievement of similar mechanical behaviour between the material and tissue, coupled with a stable interface at the connective tissue juncture. In addition, the complications of these materials were linked to their failure to maintain strength and stability during the material degradation-tissue replacement phase of healing. ${ }^{31-33}$

Requiring the resorption rate of the material to match the repair rate of the biological tissue, while ensuring that the material consists only of metabolically acceptable elements, has imposed considerable limitations on the composition, design and fabrication of these bioactive materials. ${ }^{31-33}$

Electrospinning is a technique used to synthesize continuous nanofibers from various polymers such as polycaprolactone (PCL), polyethylene oxide (PEO) and poly-L-lactic acid (PLLA). Electrospun nanofibers have been explored for use in endodontic disinfection, tooth regeneration, caries prevention, mucosal and wound repair, implant modification and tissue guided regeneration. ${ }^{34-36}$ Several limitations with electrospinning as a technique have been reported in the literature, as well as with their resultant fibres.

Electrospinning requires the use of solvents and cross linking agents for which in vivo biocompatibility may not be well established. ${ }^{37}$ Degradable synthetic polymers yield acidic by-products (during degradation) that cause inflammation in the region of their implantation. ${ }^{38}$ In addition, electrospun materials are mechanically weaker than their cast membrane counterparts and do not provide an ideal structure for dental regeneration applications requiring cellular infiltration. This is attributed to the technique yielding random unwoven mats and pore sizes, as well as not being able to fabricate micron size fibres. ${ }^{39,40}$ 
Furthermore the production efficiency of these fibres is quite low, complicating scale up production and clinical translation. ${ }^{36}$ However, the plausible advantages of electrospun scaffolds for dental therapies warrant further investment and research.

Multiple fabrication methods and technologies have been described over the years. More recent advances include additive manufacturing techniques which incorporate selective laser sintering, robocasting, stereolithography, fused deposition modelling and three dimensional (3D) printing. ${ }^{41}$ The motivation to employ such technology in Dentistry is driven by the opportunity for patient-personalized models.

At present, 3D metallic and ceramic constructs have been explored for implants, crowns and bridges using biocompatible polymers. Biological constructs including DNA plasmids, peptides, proteins and polysaccharides have also been described for use in tissue engineering therapies such as periodontal regeneration. ${ }^{42,43}$

Dental laboratories, on the other hand, have employed 3D printing for dental wax ups, orthodontic patterns, crown and bridge moulds, prosthodontic casts, etc. ${ }^{44}$ Whilst the indications for 3D printing are promising, the current laser- and inkjet-associated bioprinting techniques offer low output, with print speeds around $5 \mathrm{kHz}$, limiting manufacture to small scale. ${ }^{45}$

Furthermore, biological scaffolds are plagued by challenges facing the degradation kinetics and by the by-products of materials, due to the mass transport limitations that occur in thicker scaffolds. ${ }^{43}$ Thus, prior to encouraging the use of a 3D printed construct in clinical practice, factors such as: material costs, production volume and speed, energy costs, long-term construct performance, etc., must be evaluated and compared with traditional fabrication methods. ${ }^{46}$

\section{Laboratory studies}

\section{a. Characterization and in vitro studies}

In vitro studies involve testing of a novel material following successful fabrication and characterization. In vitro cytotoxicity tests are an essential component of biocompatibility screening, with several tests being used to ascertain the effects of a material on cell membrane integrity, cell growth and enzyme activity, or genetic expression. ${ }^{47}$

Examples of in vitro studies found in the dental implant literature include cell adhesion assays, cell proliferation assays and cell differentiation assays. ${ }^{48}$ Epithelial and fibroblast cell lineages are often chosen for biocompatibility evaluation, as ultimately these are the cells in contact with a majority of dental materials. ${ }^{49}$ Of special note here is that the cell phenotypes used in culture must match those found in vivo.

These cultured cells must be monitored not only for cell death, but for changes in their cell cycles, differentiation, and molecular biology. These are of utmost importance to account for cellular modifications that may occur following exposure to a material or device. ${ }^{50}$
Anselmo \& Mitragotri reported that whilst a plethora of preclinical studies described cellular interactions related to the introduction of inorganic nanoparticles, experimental conditions could not be normalized, leading to questionable and inconclusive claims. ${ }^{50}$ Thus, the results of biocompatibility studies must be viewed prudently especially for studies that do not acceptably simulate the oral tissue and oral environment.

Novel biomaterials require methodologies for investigating the reaction between the material and living tissues. There is no one-size-fits-all in this regard. Dental materials must account for the interactions with the tissues they encounter, which are specific to the function of the material. These include enamel, dentine, bone, periodontal ligament, and the varying oral mucosal tissues (depending on the site of action).

Materials utilized within tooth structure must be characterized according to their effects on the enamel and dentine. Those meant to bond with dentine often undergo testing for surface mechanical properties.

One such example is the modulus of elasticity which may be measured by nano-indentation tests or, by more conventional, three-points bending tests. Whilst nano-indentation boasts superior precision by quantifying changes in the submicron surface depth, it is unable to measure overall change in the dentine as a whole and is acutely affected by dentine surface roughness (which may be altered during specimen preparation). ${ }^{52}$

In order to establish an accurate biological profile for a particular material, a combination of qualitative and quantitative tests should be employed. This is necessary as, if used alone, tests may contradict one another, yielding completely different conclusions. An example of this requisite can be found in the studies by Camp et al., ${ }^{53}$ Tawil et al., ${ }^{54}$ and Torabinejad and Abu-Tahun. ${ }^{55}$

Camp compared the cell attachment properties of Mineral Trioxide Aggregate by using quantitative attachment tests with fluorescent dye. ${ }^{53}$ The same test, when later compared with in vivo histological examinations, yielded the controversial findings reported in the studies by Tawil ${ }^{54}$ and Torabinejad. ${ }^{55}$

Ding and Ma emphasized the inefficiencies with in vitro and in vivo studies in tumour research. ${ }^{56}$ They explained that the several barriers to tumour drug delivery require an in-depth understanding of the molecular mechanisms of translocations in varying $\mathrm{pH}$ environments. It is not possible to reproduce these environments without that knowledge in place. Gjorevski et al. highlighted the importance of reproducing the mechanical environment in devices functioning within the extracellular matrix and its components. ${ }^{57}$

The authors acknowledged that these environments are often difficult to establish and control in culture, in spite of recent advances in synthetic analogs and stem cell research. Hence, in the absence of this knowledge, results from in vitro studies must be interpreted with caution. 
Hench expressed concern that many materials are introduced to the clinic without lifetime prediction tests. ${ }^{31}$ He reinforced the need for standardized test methods to determine the lifetime performance of materials (specifically bioceramics) under realistic physiological stresses, including grain-boundary attack and slow crack growth. ${ }^{31,33}$

Chai et al. in their review of CaP-driven osteogenesis, highlighted the difficulties of translating intricate in vivo osteogenic environments to in vitro systems. ${ }^{58}$ The authors cautioned against the reliability of predictions and the correlation of in vitro results to what may actually occur in vivo.

Their argument was based on key issues relating to the selection of cell types and culture conditions for specific CaP-based materials. Moreover, blood vessel infiltration and body fluid composition and dynamics pose technical challenges to translation from in vitro.

Ultimately, the aim of in vitro studies should be to develop human cell-based tests that are predictable, costeffective and appropriately reliable. This will decrease the dependence of using in vivo animal tests to establish product safety, which in itself poses many ethical and economic concerns.

\section{a. In vivo studies}

In vivo studies require the selection of an appropriate animal model. The selected model should mimic human physiology as closely as possible so that appropriate conclusions can be drawn. In addition, a full description of the animal model design and evaluation (including microbiology, radiopathology and histopathology) should be available in order that the efficacy and feasibility of the material/device be established relative to current therapeutics. ${ }^{59}$

Animal ethics clearance must be obtained from the appropriate Animal Ethics Committee/s prior to initiating any animal study. Researchers may select an appropriate animal model for tests, but it is not always possible to gain approval based on the specific ethical guidelines of the relevant country. This may hinder the selection of the best suited model which at times may lead to questionable correlations and assumptions, which in turn will a ffect and delay approval for clinical trials.

Examples of animal models identified in the literature include monkeys, beagle dogs, pigs, rats and sheep. ${ }^{60}$ Furthermore, promising studies in mice and rats must also be translated to higher animals such as pigs, dogs and sheep to confirm the transitional potential of prior results. ${ }^{13}$ Pigs, in particular, share higher sequence homology with Homo sapiens making them a superior choice for animal study (as opposed to mice and rats). ${ }^{61}$

An additional challenge to in vivo studies is the requirement to reliably and reproducibly induce the same condition/s for which the experimental material/device will be indicated. An example of this necessity was explained by Jackson et al., whereby osteoradionecrosis was reliably reproduced in the mandibles of athymic rats, whilst minimizing any sources of error and variation. ${ }^{62}$
Rosa et al. demonstrated the successful regeneration of pulpal tissue when transplanting human roots in the subcutaneous tissue of mice. ${ }^{63}$ However, the authors recognized the inability of this model to completely simulate clinical conditions such as the presence of stem cells in the apical papilla. Furthermore, Guilak et al. observed that a majority of the current in vivo models simulate only normal tissue. ${ }^{64}$ Advanced disease and degenerative processes lead to major compositional and/or structural changes, which then require complex simulation models in vivo. ${ }^{64}$ Failure to achieve such environments accounts for the poor correlation of such studies to human disease.

The oral cavity poses a greater challenge to biomaterials than most other aspects of the human body. In vivo elucidation of the effects of mucosal contact, $\mathrm{pH}$ and the buffering capacity of saliva are required to negate any possible toxicity of the materials in this environment. ${ }^{15}$ In addition, materials exposed to the oral cavity are at risk of pellicle formation on their bare surfaces. Whilst there are advantages to pellicle formation, minimal colonization with pathogenic bacteria is always desired.

Pellicle formation interferes with the physicochemical surface properties of a material, and may act as a hindrance to materials possessing functions of ion and/or drug release. Furthermore, bacterial invasion may lead to infection and suboptimal tissue integration decreasing the lifespan of the device. ${ }^{65}$ Hence, low-energy surfaces are desirable to reduce these phenomena. ${ }^{66}$

Biomaterials intended for long term survival (such as dental restorations) face the challenge of water uptake, hydrolytic degradation and hygrothermal degradation. ${ }^{67-69}$ These phenomena lead to surface softening, exposing susceptible bonds which then become targets for oral enzymes, thus promoting further destruction of the material. ${ }^{70}$ Whilst water-repellant monomers have been explored, they are unsuitable due to their extensive curing time..$^{71}$ Studies have proposed an antibacterialladen monomer on the surface of oral biomaterials with the aim of preventing these challenges. However, to date, no such commercial product exists, possibly due to the augmented water uptake effect that may occur. ${ }^{72}$

In vivo studies are crucial to ascertain the effects of proposed biomaterials in a well simulated tissue environment. Researchers must strive to select and develop clinically appropriate animal models that will generate predictive data that closely correlates to human disease, which in turn will streamline the process to clinical trials.

\section{Business model}

Biomaterials have been an area of great research activity with numerous novel ideas and inventions being developed in the past decades. Yet, the number of products reaching clinical therapeutics is limited.

Whilst researchers, scientists and inventors are able rapidly to develop prototypes in their laboratories, they may at times lack the business acumen to ensure the progression of their inventions. There are several as- 
pects of the business model, each with its own hurdles, which must be fulfilled prior to clinical entry. For the purposes of this review, we have categorized them as patenting, registration, clinical trials, manufacture and scale up production. An additional factor to consider in the business model is cost. Cost will not be featured separately but will be discussed alongside every other facet of the model.

\section{a. Patenting and registration}

Novel inventions must be patented to protect the intellectual property of the inventors. Intellectual property refers to the any idea born of the intellectual creative efforts of an individual or team. It can be owned, licensed or transferred.

Forms of ownership include copyright, trademarks, utility patents, design patents, plant patents and trade secrets. This ownership is fundamental to ensuring the development and growth of a specified field, as well as granting companies expanded business and financial opportunities. ${ }^{73}$

Pressman provides useful definitions in his book "Patent It Yourself", to familiarize one's self with patents. ${ }^{74}$ An invention is "any new article, machine, composition, or process or new use developed by a human". A patent application is "a set of papers that describe an invention and that are suitable for filing in a patent office in order to apply for (or in the case of a Provisional Patent application, establish a date to apply for) a patent on the invention". Furthermore, a patent is defined as "a grant from a government that confers upon an inventor the right to exclude others from making, using, selling, importing, or offering an invention for sale for a fixed period of time". ${ }^{74}$

The regulations and laws around patenting of an invention must be well understood by the research team to ensure successful patents. Firstly, the inventor must ensure that the invention satisfies the "novelty" and "unobviousness" requirement of the Patent and Trademark Office (PTO) examiner. Second, the inventor must cognisant of the "First-to-File" law which came in to effect in March 2013.

Under the provisions of this law, should two inventors submit an application for the same patentable invention, the patent will be awarded to the person who files first, irrespective of who initially conceived and tested the invention. Exceptions are only entertained in cases where one inventor can prove the unlawful acquisition of the invention by the other inventor. Third, and of great importance, is timing. Researchers must register a patent application to secure their intellectual property prior to any research publications.

This refers not only to journal publications, but any form of conference or congress proceedings, meetings, etc. Once the invention is made public, it loses novel status and the patenting process is placed in jeopardy.

The only country that grants a one-year grace period for the submission of a patent application following public disclosure, is the United States of America.
An additional factor the inventor must be mindful of is that the registration of a patent is region and/or country specific, with no mutually agreed standardised guidelines. Some countries, like South Africa, allow for non-examinable patents whereas others, such as the US Patents and Trademarks Office (USPTO) and European Patents Office (EPO), examine patents prior to granting them. The disadvantage of a non-examined patent is that errors and over-extended claims may go undetected.

Patents that have unjustifiably broad claims also hinder future research and development in the field. Some inventions may not even meet patentable criteria ('subpatentable inventions'). ${ }^{75}$ The research team must identify all of the countries in which they wish to register their patent and apply as required. The researcher may then pursue publication once the patent application has been successfully filed.

There are two types of patent applications: a Provisional Patent Application (PPA) and a Regular Patent Application (RPA). The benefit of a PPA is that it grants an earlier filing date prior to building and testing an invention and does not count toward the 20 year patent term. However, no amendments or additions may be made to the PPA once filed and the earlier filing date is only valid if the description of the invention is legally sufficient.

The inventors must bear in mind that PPA's are not examined, have no legal grounds other than the filing date, and require the RPA be filed within one year following the initial PPA. ${ }^{74}$ Figure 2 outlines the process for obtaining a utility patent as described by the USPTO on their official website. ${ }^{76}$

Recent partnerships between academic laboratories and pharmaceutical companies have augmented fundamental drug delivery research, patenting and registration and the resultant translation to improved clinical therapeutics.

Once a prototype is patented and, and preclinical studies confirm its function and value, inventors will look to the US Food and Drug Administration (FDA) for regulatory approval. The FDA poses an enormous barrier to the clinical translation of new materials and devices.

The FDA's Office of Medical Products and Tobacco houses three centres of regulation; namely: the Centre for Drug Evaluation and Research (CDER), the Centre for Biologic Evaluation and Research (CBER), and the Centre for Devices and Radiological Health (CDRH). New materials must pass through clinical trials, which often extend over a 6-12 year testing and review period, involving thousands of patients with resultant costs escalating to the hundreds of millions of dollars.

Start-up companies, funded by venture capitalists, are at their highest risk during this process. The risk of inventions failing to gain FDA approval, coupled with poor initial sales (failing to overcome production costs) are often factors cited for the shutting down of small companies. One such example is the company Organogenesis (Canton, MA) who launched Apligraf Ò skin equivalent. 


\section{Step Who? Activity}

1.

\section{Applicant}

Has your invention already been patented? Search: http//patft.uspto.gov Yes End

2. Applicant

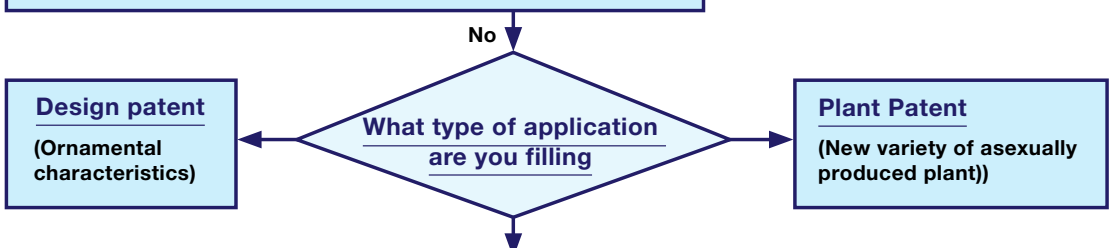

3.

\section{Applicant}

Utility patent (most common)

(Useful process, machine, article of manufacture, composition of matter)

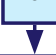

4.

\section{Applicant}

$\checkmark$

5.

\section{Applicant}

6.

\section{Applicant}

7.

\section{Applicant}

8.

\section{Applicant}

9.

USPTO

10.

\section{Applicant}

11.

\section{USPTO}

12.

\section{Applicant}

13.

\section{Applicant}
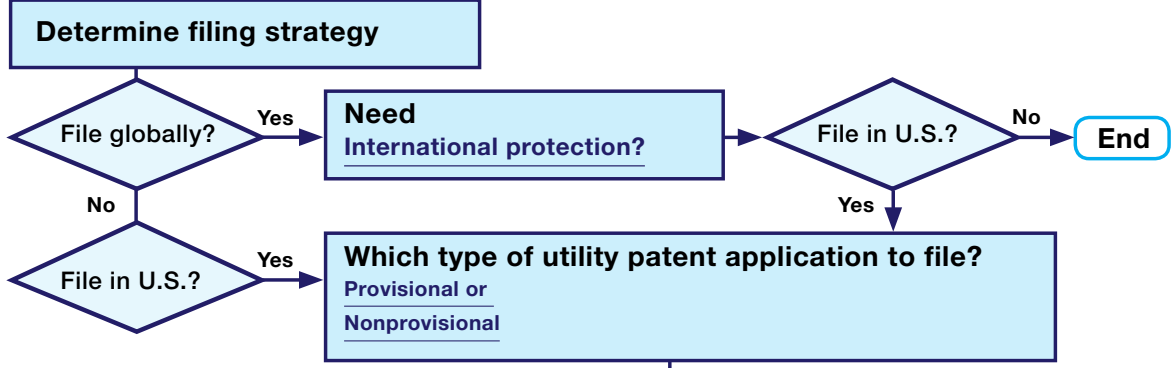

Which type of utility patent application to file?

Provisional or

Nonprovisional

\section{Consider expedited examination}

Prioritized examination

Accelerated examination program

First action interview

Patents prosecution highway

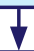

\section{Who should file? \\ File yourself (pro se) \\ Use a registered attorney or agent (recommended)}

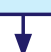

\section{Prepare for electronic filing}

Determine application processing fees

Apply for a customer number and digital certificate

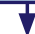

Apply for patent using Electronic Filing System

as a registered eFiler (recommended)

About EFS-Web

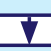

\section{USPTO examines application}

Check application status

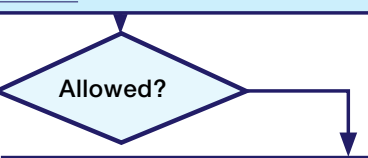

Applicant files replies, requests for reconsideration, and appeals as necessary

If objections and rejection of the examiner are overcome, USPTO sends Notice of Allowence and Fee(s) due

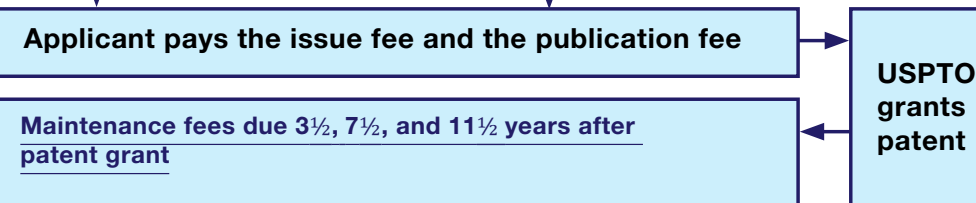


Whilst the product gained regulatory approval, market sales failed to achieve profits leading to the company filing for bankruptcy in 2001. ${ }^{77}$ Lysaght et al. further described 18 other companies that either filed for bankruptcy or simply closed their doors following devastating results from their clinical trials, combined with irredeemable financial losses. ${ }^{77}$ Hence, both new and existing companies must take into account that the uptake of their product in the market will be gradual. Whilst high profits may be attained, but in all likelihood that will be over a period of several years.

\section{b. Clinical trials}

Following successful in vitro and in vivo tests that establish manufacturing standardization, efficacy and safety of an invention, ethical clearance is acquired for human clinical trials. The main objective of clinical trials is to confirm the safety and efficacy of the invention in humans. These are then followed by an application for regulatory approval of commercial sales of the invention which is submitted to the appropriate regulatory authority, for example, the FDA.

Anselmo and Mitragotri aligned the success of clinical translation of an invention to it being safe, performing its desired function, offering convenient administration and easy fabrication. ${ }^{51}$ Whilst numerous inventions are explored at laboratory level, few develop to clinical products.

The failure to obtain regulatory approval for clinical trials may be attributed to limited in vitro and in vivo data, and perhaps short-sightedness on the part of inventors in not completely understanding the biological mechanisms of the regions in which the invention will be applicable. ${ }^{78} \mathrm{~A}$ restriction of clinical trials is that study samples are limited to tissue biopsies, swabs or fluid aspirates. Animal (in vivo) studies, on the other hand, allow for endpoint microbiological and histological examinations of the tissue, enabling more rigorous analysis. ${ }^{59}$

Regulatory approval requires a satisfactory set of preclinical data backed up by equivalent in vivo (animal) data. A large quantity of animal data may at times be required, which escalates costs. In addition, the clinical trial in itself poses a severe cost factor which is difficult to predict. One such prediction required for approval is survivability, which for inventions such as implants, must be established for $3-5$ years. ${ }^{33}$

French et al. explored the use of human pluripotent stem cells to address unmet clinical needs. ${ }^{79}$ They identified two companies, Geron Corporation (Menlo Park, California) and Advance Cell Technology Inc. (Santa Monica, California), who were the first to evaluate the safety of embryonic stem cells in clinical (patient) trials.

Whilst not all of these trials ran until completion, the exercise proved educational for the researchers and for the regulatory authority. The authors noted several regulatory challenges to the clinical translation of stem cells (depicted in Figure 3) and concluded that uncontrolled irreproducibility and variability were the key factors hindering clinical therapeutics.

\section{c. Manufacture and scale up production}

Reaching this stage of the business model sheds first light on clinical entry. No doubt, the monetary investment of the invention has surpassed millions at this stage and it is in the interest of the investors to push forward. At this juncture, the calculation of potential cost/profit and risk/reward ratios are not yet possible and the decision to proceed is based on the confidence of all those concerned.

Furthermore, the company launching the products needs to survive through to the realisation of profit. The phase from breaking even to realizing acceptable profit margins must be accelerated and is frequently a challenge for smaller businesses. However, money aside, the manufacture and scale up process comes with its own challenges.

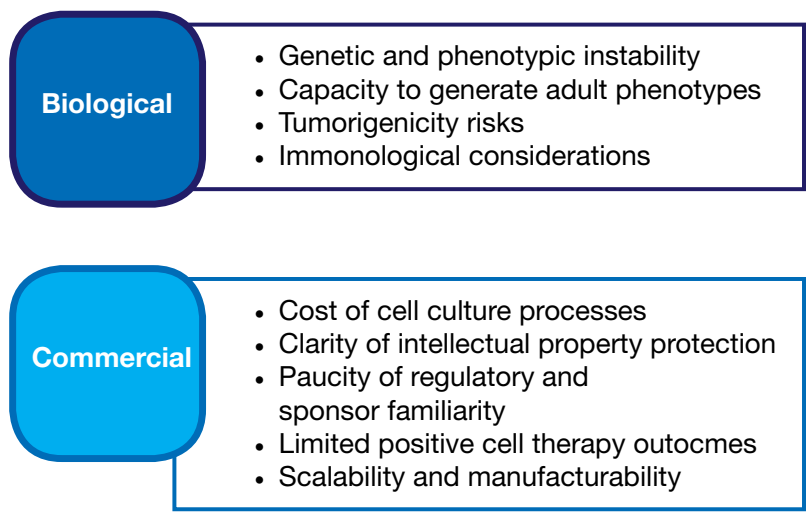

Figure 3. Regulatory challenges to successful clinical translation of stem cells (adapted from French et al., 2015)

Laboratory synthesized inventions face severe engineering challenges in their scale up to safe, reproducible, clinically effective, and economically stable designs. ${ }^{80}$

The sale volumes of inventions are dependent on the cost of production and the resultant price to consumer. These prices must be within an acceptable range to ensure buy-in from the market and consequent patient benefit. Furthermore, inventions that require additional procedures (such as surgery) may initially be frowned upon unless the market demand is high and the invention is replacing alternatives that are more expensive. ${ }^{81}$

A plethora of inventions are usually synthesized within laboratories, by researchers and scientists who themselves drive the processes. These manual methods of fabrication make scaling up difficult. One such example is cell-based therapies that rely on manual seeding and culturing of scaffolds, which is inefficient, time-consuming and operator dependent. ${ }^{82}$

An opportunity presents here for synergistic collaboration between large companies and scientists to remodel and automate fabrication methods that ensure successful scale up. This requires the development of reproducible and consistent techniques that will optimise manufacturing processes, and enable an increase in production volumes whilst reducing costs. 
Schiller et al. (2015) described a novel focused ultrasound technique to manufacture nanoparticles which could possibly serve as a tool to enhance clinical translation of such particles. ${ }^{83}$ Additive Manufacturing (AM), also known as rapid prototyping, is another example of a developed technique that guarantees standardisation of the manufacturing process, and allows for the fabrication of materials that meet stringent performance criteria for clinical use, whilst offering the flexibility to scale up fabrication. ${ }^{30}$

Ramshaw et al. stressed that straightforward purification processes are required to display the scalability of products, whilst Langer and Peppas also highlighted the issue of immunogenicity and purification (of contaminants) during large scale production. ${ }^{84,85}$ Strategies for the preservation, packaging and distribution of the invention must also be developed at this stage due to their financial impact on the final product. ${ }^{64,81}$ Luong et al. demonstrated dry storage strategies for bioactive hydrogels that yielded adequate shelf life, when appropriate stabilization was employed. ${ }^{86}$ It is worthwhile considering and improving these factors prior to the application for clinical trials. Clinical trials pose a major financial burden on the company, and further escalate the price of the emerging product.

The constituent materials of an invention may also affect the manufacturing process. A reproducible invention requires consistent properties of the constituent materials. Unfortunately, due to the differing production processes between the various suppliers, companies may be left with no choice but to select a sole supplier. This introduces an additional risk factor to the manufacturing process, in that suppliers may raise material costs, or unexpectedly close down. ${ }^{51}$ While a brief explanation of natural and synthetic polymers has already been given, it is necessary at this point to discuss some of the reservations around the use of natural materials.

The natural variability of animal tissue preparations has led to concerns regarding their purity, predictability of performance, and the possibility that disease transmission that may occur. ${ }^{84}$ In addition, though having been proven to be safe and effective, some natural materials (such as bovine collagen) have indeed induced adverse immune reactions in a small percentage of patients. ${ }^{87}$ These shortfalls have been countered by more recent advances in genetic recombinant technology which offers scientific superiority in designing biologically complex structures.

Other advantages include standardized product quality and ease of isolation and purification with no risk of cross infection. However, the initial setback of this technology is that a thorough knowledge of the genetic sequence is required before it can be employed. ${ }^{88}$ Furthermore, commercial scale production of recombinant technology is more difficult to achieve and requires evaluation of the manufacturing and economical processes. ${ }^{81} \mathrm{~A}$ good example to demonstrate the importance of these mechanisms is the product Nutropin $\AA$, a recombinant growth hormone for paediatric application.
The product acquired FDA approval in 1999 and was marketed by Genentech. However, Nutropin $₫$ was discontinued in 2004 for reasons associated with its high costs and protracted manufacturing process, two weeks being required to synthesise each batch. ${ }^{89}$

\section{Clinical application}

Accomplishing clinical entry is a great achievement for any material or device. No doubt, by this time, millions have been invested in the product and the investors will be anxious about the market response. However, the success of previously introduced novel products may offer some solace. One such example is Abraxane $\AA$, for which the company reported a 52 percent year-on-year increase with a value of $\$ 649$ million. ${ }^{90}$

One of the final challenges to clinical entry is to gain buy-in from the end users. Clinicians are often resistant to change and will be unlikely to try a new product in the absence of quality assurance. This poses a challenge to companies introducing novel inventions in that the data displayed by the company is often seen as biased, with clinicians looking to other sources for confirmation of the proposed claims for the inventions. Studies in which a conflict of interest exists are commonly distrusted as the authors are supposedly seen as biased toward the product. One approach of the dental companies has been to approach university faculty staff to assess the claims of an invention. This has led to independent research that either confirms or refutes the claims of the product and which seeks to confirm reproducible results whilst satisfying the required clinical criteria. Padovani et al. suggested that for dental restorative materials, the important factors for consideration must be the results from long-term studies, post-operative sensitivity, retention of prosthodontic restorations and marginal sealing ability. ${ }^{15}$ Thus, further clinical studies are always recommended to augment understanding of the mechanism of action of each material within the dynamic oral cavity.

Additional factors to consider are cost and product comparison. Novel products must outperform their existing counterparts, at a competitive cost not considerably greater, if they are to be accepted by clinicians. Companies often approach leading clinicians in a field to test their inventions (free of charge).

Should the chosen clinicians provide positive feedback, they are invited to become the face of the new product to drive acceptability and incorporation of the novel material or device in to clinical treatment modalities.

\section{CONCLUSION}

In 1987, Williams defined a biomaterial as a nonviable material used in a medical device, intended to interact with biological systems. ${ }^{91}$ Material science has come a long way since then and newer definitions are being offered with every passing year to account for novel advances in clinical medicine. 
A substantial part of the literature still reports five fundamental classes of biomaterials, namely: natural materials, polymers, metals, bioceramics and composites. However, the boundaries of these individual classes have blurred with combinant technology that draws on the strength of differing materials to yield innovations of superior character.

A deep understanding of the product development process is mandatory when conceptualizing novel ideas. It is imperative that the end-goal of clinical translation be borne in the minds of researchers when they contemplate the materials, designs and fabrication methods for novel inventions. A thorough understanding of the biological complexities found in both physiological and pathological environments enable the design of appropriately suited dental biomaterials. The limitations of manual production methods should also be recognized, with an emphasis placed on automated manufacturing processes to assist product scale up. Utilizing approved constituent materials in conjunction with standardized fabrication methods are examples of strategies which are effective in accelerating the development process.

The commercial viability of products must be gauged at the point of invention to protect intellectual property, following approval by regulatory authorities. In addition, a substantial monetary investment is required for the successful translation of novel products. This burden remains a major hurdle for start-up companies, who cannot always absorb the delay in market response.

Whilst several obstacles to successful clinical translation have been highlighted in this review, none are beyond resolution. Collaborations must be encouraged between clinicians and scientists that may yield discussions which will cultivate innovative strategies for improved and enhanced patient care. Biomaterial science is a crucial element in the advance of all clinical disciplines.

\section{References}

1. Tathe A, Ghodke M, Nikalje AP. A brief review: biomaterials and their application. International Journal of Pharmacy and Pharmaceutical Sciences 2010; 2: 19-23.

2. Holzapfel BM, Reichert JC, Schantz J-T, Gbureck U, Rackwitz L, Nöth U, Jakob F, Rudert M, Groll J, Hutmacher DW. How smart do biomaterials need to be? A translational science and clinical point of view. Advanced Drug Delivery Reviews 2013; 65: 581-603.

3. Parida P, Behera A, Mishra SC. Classification of Biomaterials used in Medicine. 2012. [accessed 2016 Mar 1]. http://dspace. nitrkl.ac.in/dspace/handle/2080/1761.

4. Ramakrishna S, Mayer J, Wintermantel E, Leong KW. Biomedical applications of polymer-composite materials: a review. Composites Science and Technology 2001; 61: 1189-224.

5. Lane WA. Some remarks on the treatment of fractures. British Medical Journal 1895; 1: 861.

6. Leek FF. The practice of Dentistry in Ancient Egypt. The Journal of Egyptian Archaeology 1967; 53: 51.

7. Hermawan H, Ramdan D, Djuansjah JR. Metals for biomedical applications. INTECH Open Access Publisher 2011. [accessed 2016 Feb 3]. http://www.intechopen.com/source/ pdfs/18658/InTech-Metals_for_biomedical_applications.pdf.

8. Mandracci P, Mussano F, Rivolo P, Carossa S. Surface treatments and functional coatings for biocompatibility improvement and bacterial adhesion reduction in dental implantology. Coatings 2016; 6:7.
9. Sáenz A, Rivera E, Brostow W, Castaño VM. Ceramic biomaterials: an introductory overview. Journal of Materials Education 1999; 21(5/6): 267-76.

10. Dorozhkin SV. Calcium orthophosphate bioceramics. Ceramics International 2015; 41: 13913-66.

11. Ratner BD, editor. Biomaterials Science: an Introduction to Materials in Medicine. 2004; 2nd ed. Amsterdam; Boston: Elsevier Academic Press.

12. Bao Ha TL, Minh T, Nguyen D, Minh D. Naturally derived biomaterials: Preparation and application. In: Andrades $\mathrm{JA}$, editor. Regenerative Medicine and Tissue Engineering. InTech. 2013 [accessed 2016 Feb 3]. http://www.intechopen. com/books/regenerative-medicine-and-tissue-engineering/ naturally-derived-biomaterials-preparation-and-application

13. Agarwal R, García AJ. Biomaterial strategies for engineering implants for enhanced osseointegration and bone repair. Advanced Drug Delivery Reviews 2015; 94: 53-62.

14. Neel EA, Bozec L, Perez RA, Kim HW, Knowles JC. Nanotechnology in Dentistry: prevention, diagnosis, and therapy. International Journal of Nanomedicine 2015; 10: 6371.

15. Padovani GC, Feitosa VP, Sauro S, Tay FR, Durán G, Paula AJ, Durán N. Advances in dental materials through nanotechnology: Facts, perspectives and toxicological aspects. Trends in Biotechnology 2015; 33: 621-36.

16. Dorozhkin SV, Epple M. Biological and medical significance of calcium phosphates. Angewandte Chemie International Edition in English 2002; 41: 3130-46.

17. Miron RJ, Zhang Q, Sculean A, Buser D, Pippenger BE, Dard M, Shirakata Y, Chandad F, Zhang Y. Osteoinductive potential of four commonly employed bone grafts. Clinical Oral Investigations 2016: 1-7.

18. Swetha M, Sahithi K, Moorthi A, Srinivasan N, Ramasamy K, Selvamurugan N. Biocomposites containing natural polymers and hydroxyapatite for bone tissue engineering. International Journal of Biological Macromolecules 2010; 47: 1-4.

19. Anselmo AC, Mitragotri S. An overview of clinical and commercial impact of drug delivery systems. Journal of Controlled Release 2014; 190: 15-28.

20. Srakaew V, Ruangsri P, Suthin K, Thunyakitpisal P, Tachaboonyakiat W. Sodium-phosphorylated chitosan/zinc oxide complexes and evaluation of their cytocompatibility: an approach for periodontal dressing. Journal of Biomaterials Applications 2011: 0885328211408371.

21. Shaikh RP, Pillay V, Choonara YE, du Toit LC, Ndesendo VMK, Bawa P, Cooppan S. A review of multiresponsive membranous systems for rate-modulated drug delivery. AAPS Pharm Sci Tech. 2010; 11: 441-59.

22. Santos A, Sinn Aw M, Bariana M, Kumeria T, Wang Y, Losic D. Drug-releasing implants: current progress, challenges and perspectives. Journal of Materials Chemistry B 2014; 2: 6157.

23. Moghanjoughi AA, Khoshnevis D, Zarrabi A. A concise review on smart polymers for controlled drug release. Drug Delivery and Translational Research 2016: 1-8.

24. Gandhi A, Paul A, Sen SO, Sen KK. Studies on thermoresponsive polymers: Phase behaviour, drug delivery and biomedical applications. Asian Journal of Pharmaceutical Sciences 2015; 10: 99-107.

25. Fleige E, Quadir MA, Haag R. Stimuli-responsive polymeric nanocarriers for the controlled transport of active compounds: concepts and applications. Advanced Drug Delivery Reviews 2012; 64: 866-84.

26. Hearnden V, Sankar V, Hull K, Juras DV, Greenberg M, Kerr AR, Lockhart PB, Patton LL, Porter S, Thornhill MH. New developments and opportunities in oral mucosal drug delivery for local and systemic disease. Advanced Drug Delivery Reviews 2012; 64: 16-28.

27. Boddupalli B, Mohammed Z., Nath R, Banji D. Muco-adhesive drug delivery system: An overview. Journal of Advanced Pharmaceutical Technology and Research 2010; 1: 381. 
28. Mansuri S, Kesharwani P, Jain K, Tekade RK, Jain NK. Mucoadhesion: a promising approach in drug delivery system. Reactive and Functional Polymers 2016; 1; 100: 151-72.

29. León B, Jansen J, editors. Thin Calcium Phosphate Coatings for Medical Implants. 2009 New York, NY: Springer New York. [accessed $2016 \mathrm{Apr}$ 15]. http://link.springer.com/10. 1007/978-0-387-77718-4.

30. Tang D, Tare RS, Yang LY, Williams DF, Ou KL, Oreffo RO. Biofabrication of bone tissue: approaches, challenges and translation for bone regeneration. Biomaterials. 2016; 83: 363-82.

31. Hench LL. Bioceramics: from concept to clinic. Journal of the American Ceramic Society 1991; 74: 1487-510.

32. Hench LL, Hench JW, Greenspan DC. Bioglass: a short history and bibliography. Journal of the Australasian Ceramic Society 2004; 40: 1-42.

33. Hench LL, Jones JR. Bioactive Glasses: Frontiers and Challenges. Frontiers in Bioengineering and Biotechnology 3 2015 [accessed 2016 Feb 3]. http://journal.frontiersin.org/ Article/10.3389/fbioe.2015.00194/abstract.

34. Bottino MC, Kamocki K, Yassen GH, Platt JA, Vail MM, Ehrlich Y, Spolnik KJ, Gregory RL. Bioactive nanofibrous scaffolds for regenerative endodontics. Journal of Dental Research 2013; 92: 963-9.

35. Albuquerque MT, Valera MC, Nakashima M, Nör JE, Bottino MC. Tissue-engineering-based strategies for regenerative endodontics. Journal of Dental Research. 2014; 93(12): 1222-31.

36. Zafar M, Najeeb S, Khurshid Z, Vazirzadeh M, Zohaib S, Najeeb B, Sefat F. Potential of electrospun nanofibers for biomedical and dental applications. Materials 2016; 9(2): 73.

37. Tamimi E, Ardila DC, Haskett DG, Doetschman T, Slepian MJ, Kellar RS, Vande Geest JP. Biomechanical comparison of glutaraldehyde-crosslinked gelatin fibrinogen electrospun scaffolds to porcine coronary arteries. Journal of Biomechanical Engineering 2016; 138: 011001.

38. Sheikh Z, Najeeb S, Khurshid Z, Verma V, Rashid H, Glogauer M. Biodegradable materials for bone repair and tissue engineering applications. Materials 2015; 8: 5744-94.

39. Guimarães A, Martins A, Pinho ED, Faria S, Reis RL, Neves NM. Solving cell infiltration limitations of electrospun nanofiber meshes for tissue engineering applications. Nanomedicine (Lond) 2010; 5: 539-54.

40. Gupte MJ, Ma PX. Nanofibrous scaffolds for dental and craniofacial applications. Journal of Dental Research 2012; 91: 227-34.

41. Mota C, Puppi D, Chiellini F, Chiellini E. Additive manufacturing techniques for the production of tissue engineering constructs: Journal of Tissue Engineering and Regenerative Medicine 2015; 9: 174-190.

42. Chia HN, Wu BM. Recent advances in 3D printing of biomaterials. Journal of Biological Engineering 2015; 9: 4.

43. Larsson L, Decker AM, Nibali L, Pilipchuk SP, Berglundh T, Giannobile WV. Regenerative medicine for periodontal and peri-implant diseases. Journal of Dental Research. 2016; 95(3): 255-66.

44. Stansbury JW, Idacavage MJ. 3D printing with polymers: Challenges among expanding options and opportunities. Denta Materials 2016; 32: 54-64.

45. Guillotin B, Catros S, Keriquel V, Souquet A, Fontaine A, Remy M, Fricain J. Rapid prototyping of complex tissues with laser assisted bioprinting ( $L A B)$. Rapid Prototyping of Biomaterials: Principles and Applications 2014: 156.

46. Weller C, Kleer R, Piller FT. Economic implications of 3D printing: Market structure models in light of additive manufacturing revisited. International Journal of Production Economics 2015; 164: 43-56.

47. Ahmed HMA, Luddin N, Kannan TP, Mokhtar KI, Ahmad A. Cell attachment properties of Portland cement-based endodontic materials: biological and methodological considerations. Journal of Endodontics 2014; 40: 1517-23.
48. Anitua E, Piñas L, Murias A, Prado R, Tejero R. Effects of calcium ions on titanium surfaces for bone regeneration. Colloids and Surfaces B: Biointerfaces 2015; 130: 173-81.

49. Faria AC, Rodrigues RC, Rosa AL, Ribeiro RF. Experimental titanium alloys for dental applications. The Journal of Prosthetic Dentistry 2014; 112: 1448-60.

50. Hench LL, Thompson I. Twenty-first century challenges for biomaterials. Journal of the Royal Society Interface 2010; 7 : S379-S391.

51. Anselmo AC, Mitragotri S. A review of clinical translation of inorganic nanoparticles. The AAPS Journal 2015; 17: 1041-54.

52. Fawzy AS, Nitisusanta LI, lqbal K, Daood U, Beng LT, Neo J. Chitosan/Riboflavin-modified demineralized dentin as a potential substrate for bonding. Journal of the Mechanical Behavior of Biomedical Materials 2013; 17: 278-89.

53. Camp MA, Jeansonne BG, Lallier T. Adhesion of human fibroblasts to root-end-filling materials. Journal of Endodontics 2003; 29: 602-7.

54. Tawil PZ, Trope M, Curran AE, Caplan DJ, Kirakozova A, Duggan DJ, Teixeira FB. Periapical microsurgery: an in vivo evaluation of endodontic root-end filling materials. Journal of Endodontics 2009; 35: 357-62.

55. Torabinejad M, Abu-Tahun I. Management of teeth with necrotic pulps and open apices. Endodontic Topics 2010; 23: 105-30.

56. Ding $\mathrm{H}$, Ma Y. Controlling cellular uptake of nanoparticles with $\mathrm{pH}$-sensitive polymers. Scientific reports 3, 2013 [accessed 2016 Feb 26]. http://www.nature.com/srep/2013/130930/ srep02804/full/srep02804.html.

57. Gjorevski N, Ranga A, Lutolf MP. Bioengineering approaches to guide stem cell-based organogenesis. Development 2014; 141: 1794-1804

58. Chai YC, Carlier A, Bolander J, Roberts SJ, Geris L, Schrooten J, Van Oosterwyck H, Luyten FP. Current views on calcium phosphate osteogenicity and the translation into effective bone regeneration strategies. Acta Biomaterialia 2012; 1; 8(11): 3876-87.

59. Inzana JA, Schwarz EM, Kates SL, Awad HA. Biomaterials approaches to treating implant-associated osteomyelitis. Biomaterials 2016; 81: 58-71.

60. Bright R, Hynes K, Gronthos S, Bartold PM. Periodontal ligament-derived cells for periodontal regeneration in animal models: a systematic review. Journal of Periodontal Research 2015; 50: 160-72.

61. Landesman-Milo D, Peer D. Transforming nanomedicines from lab scale production to novel clinical modality. Bioconjugate Chemistry 2016 [accessed 2016 Mar 1]. http:// pubs.acs.org/doi/10.1021/acs.bioconjchem.5b00607.

62. Jackson RS, Voss SG, Wilson ZC, Remmes NB,

63. Stalboerger PG, Keeney MG, Moore EJ, Janus JR. An athymic rat model for mandibular osteoradionecrosis allowing for direct translation of regenerative treatments. Otolaryngology - Head and Neck Surgery 2015; 153: 526-31.

64. Rosa V, Della Bona A, Cavalcanti BN, Nör JE. Tissue engineering: from research to dental clinics. Dental Materials. 2012; 28(4): 341-8.

65. Guilak F, Butler DL, Goldstein SA, Baaijens FPT. Biomechanics and mechanobiology in functional tissue engineering. Journal of Biomechanics 2014; 47: 1933-40.

66. Hench LL, Thompson I. Twenty-first century challenges for biomaterials. Journal of the Royal Society Interface. 2010; 19; 7(suppl_4): S379-91.

67. Hannig C, Hannig M. The oral cavity - a key system to understand substratum-dependent bioadhesion on solid surfaces in man. Clinical Oral Investigations 2009; 13: 123-39.

68. Mayworm CD, Camargo SS, Bastian FL. Influence of artificial saliva on abrasive wear and microhardness of dental composites filled with nanoparticles. Journal of Dentistry 2008; 36: 703-10. 
69. Reis A, Ferreira SQ, Costa TRF, Klein-Júnior CA, Meier MM, Loguercio AD. Effects of increased exposure times of simplified etch-and-rinse adhesives on the degradation of resindentin bonds and quality of the polymer network: Polymerization and adhesive durability. European Journal of Oral Sciences 2010; 118: 502-9.

70. Bertassoni LE, Orgel JPR, Antipova O, Swain MV. The dentin organic matrix - limitations of restorative dentistry hidden on the nanometer scale. Acta Biomaterialia 2012; 8: 2419-33.

71. Li Y, Carrera C, Chen R, Li J, Lenton P, Rudney JD, Jones RS, Aparicio C, Fok A. Degradation in the dentin-composite interface subjected to multi-species biofilm challenges. Acta Biomaterialia 2014; 10: 375-83.

72. Kadoma Y. Kinetic polymerization behavior of fluorinated monomers for dental use. Dental Materials Journal 2010; 29: 602-8.

73. Delaviz Y, Finer $Y$, Santerre JP. Biodegradation of resin composites and adhesives by oral bacteria and saliva: A rationale for new material designs that consider the clinical environment and treatment challenges. Dental Materials 2014; 30: 16-32.

74. Brougher JT. 2014. Introduction to intellectual property. In: Brougher TJ, editor. Intellectual Property and Health Technologies: Balancing Innovation and the Public's Health. New York, NY: Springer New York. 2014: 1-18.

75. Pressman D. Patent It Yourself: Your Step-by-Step Guide to Filing at the U.S. Patent Office. Nolo 2014.

76. Ncube CB. The draft National Intellectual Property Policy proposals for improving South Africa's patent registration system: a review. Journal of Intellectual Property Law \& Practice 2014; 9: 822-9.

77. USPTO. Process for Obtaining a Utility Patent. 2015 [accessed 2016 Mar 4]. http://www.uspto.gov/patents-getting-started/patent-basics/types-patent-applications/utility-patent/ process-obtaining.

78. Lysaght MJ, Jaklenec A, Deweerd E. Great expectations: private sector activity in tissue engineering, regenerative medicine, and stem cell therapeutics. Tissue Engineering Part A. $2008 ; 14(2): 305-15$.

79. Miguez-Pacheco V, Hench LL, Boccaccini AR. Bioactive glasses beyond bone and teeth: emerging applications in contact with soft tissues. Acta Biomaterialia 2015; 13: 1-15.

80. French A, Bravery C, Smith J, Chandra A, Archibald P, Gold JD, Artzi N, Kim H-W, Barker RW, Meissner A, et al. Enabling consistency in pluripotent stem cell-derived products for research and development and clinical applications through material standards. Stem Cells Translational Medicine 2015; 4: 217-23.
81. Naughton GK. From lab bench to market. Annals of the New York Academy of Sciences 2002; 961: 372-85.

82. Williams DJ, Sebastine IM. Tissue engineering and regenerative medicine: manufacturing challenges. IEE Proceedings - Nanobiotechnology 2005; 152: 207.

83. Ferris CJ, Gilmore KG, Wallace GG, others. Biofabrication: an overview of the approaches used for printing of living cells. Applied Microbiology and Biotechnology 2013; 97: 4243-58.

84. Schiller S, Hanefeld A, Schneider M, Lehr C-M. Focused ultrasound as a scalable and contact-free method to manufacture protein-loaded plga nanoparticles. Pharmaceutical Research 2015; 32: 2995-3006.

85. Ramshaw JA, Werkmeister JA, Dumsday GJ. Bioengineered collagens: Emerging directions for biomedical materials. Bioengineered 2014; 5: 227-33.

86. Langer R, Peppas NA. Advances in biomaterials, drug delivery, and bionanotechnology. American Institute of Chemical Engineers Journal 2003; 49: 2990-3006.

87. Luong PT, Browning MB, Bixler RS, Cosgriff-Hernandez E. 2014. Drying and storage effects on poly (ethylene glycol) hydrogel mechanical properties and bioactivity. Journal of Biomedical Materials Research Part A, 2014; 102: 3066-76.

88. Alijotas-Reig J, Fernández-Figueras MT, Puig L. Inflammatory, immune-mediated adverse reactions related to soft tissue dermal fillers. In: Seminars in Arthritis and Rheumatism, 2013; 43(2): 241-258.

89. WB Saunders, Werkmeister JA, Ramshaw JA. Recombinant protein scaffolds for tissue engineering. Biomedical Materials 2012; 7: 012002.

90. Jordan F, Naylor A, Kelly CA, Howdle SM, Lewis A, Illum L. Sustained release $\mathrm{hGH}$ microsphere formulation produced by a novel supercritical fluid technology: In vivo studies. Journal of Controlled Release 2010; 141: 153-60.

91. Celgene Corporation Announces 2014 Financial Outlook and Preliminary 2013 Results (NASDAQ:CELG). [accessed 2016b Mar 2]. http://ir.celgene.com/releasedetail.cfm?Release $\mathrm{ID}=821044$.

92. Williams DF. Definitions in Biomaterials: Proceedings of a Consensus Conference of the European Society for Biomaterials, Chester, England, March 3-5, 1986. 1987 Elsevier Science Limited.

\section{Do the CPD questionnaire on page 53}

The Continuous Professional Development (CPD) section provides for twenty general questions and five ethics questions. The section provides members with a valuable source of CPD points whilst also achieving the objective of CPD, to assure continuing education. The importance of continuing professional development should not be underestimated, it is a career-long obligation for practicing professionals.

\section{Online CPD in 6 Easy Steps}

Go to the SADA website www.sada.co.za

2 Log into the 'member only' section with your unique SADA username and password.

3 Select the CPD navigation tab.

4 Select the questionnaire that you wish to complete.

5 Enter your multiple choice answers. Please note that you have two attempts to obtain at least $70 \%$.

6 View and print your CPD certificate. 